\title{
Síntese e caracterização de perovskitas LaMnO3 e $Y$-alumina como catalisadores para a reação de combustão do metano
}

\section{Synthesis and characterization of perovskites $\mathrm{LaMnO}_{3}$ and $\mathrm{y}$-alumina as catalysts for methane combustion reaction}

\author{
Vanessa Pereira Gonzaga Santos ${ }^{1}$, Milena de Santana Santos ${ }^{1}$,
} Carlo Resini ${ }^{2}$, Soraia Teixeira Brandão ${ }^{1}$

\footnotetext{
${ }^{1}$ Grupo de Catálise e Polímeros - GCP - IQ/PPEP/UFBA,Campus de Ondina CEP: 40170-115, Salvador, BA e-mail: pgs.vanessa@gmail.com

${ }^{2}$ International Iberian Nanotechnology Laboratory, Braga, 4715-330, Portugal

e-mail: anelim_75@hotmail.com; soraia.ufba@gmail.com; carlo.resini@gmail.com
}

\section{RESUMO}

Catalisadores do tipo perovskita, $\mathrm{LaMnO}_{3}$, foram sintetizados pelos métodos citrato e coprecipitação. Parte destes materiais foi suportado em $\gamma$-alumina ou impregnados por via úmida com $\mathrm{Pd}$ e/ou $\mathrm{Sn}$ na concentração de $1 \%$ em massa. Os catalisadores foram avaliados na reação de combustão total do metano, tendo a sua atividade medida através de reação superficial termoprogramada (TPSR). Para tanto, as amostras foram aquecidas até $800^{\circ} \mathrm{C}$, a uma taxa de $10^{\circ} \mathrm{C} / \mathrm{min}$, sob um fluxo de $50 \mathrm{~mL} / \mathrm{min}$ de uma mistura contendo $\mathrm{CH}_{4}, \mathrm{O}_{2}$ e $\mathrm{N}_{2}$ com concentrações molares de $4 \%, 1 \%$ e $95 \%$, e He $(50 \mathrm{~mL} / \mathrm{min})$. Como padrão de comparação, sintetizou-se também uma amostra de referência de $\mathrm{PdO} / \gamma-\mathrm{Al}_{2} \mathrm{O}_{3}$. As amostras foram caracterizadas por análise termogravimétrica (ATG), difração de raios X (DRX) e tiveram a sua área superficial medida por BET. As amostras contendo paládio foram ainda analisadas na reação de oxidação a temperatura programada (TPO) de modo a determinar a estabilidade das fases PdO presentes nestes materiais. Todas as amostras se apresentaram ativas para a reação de combustão do metano, sendo os materiais oriundos da rota citrato superiores aos materiais coprecipitados. A adição de paládio, mesmo em baixa concentração, mostrou-se positiva para a atividade catalítica, ao contrário do estanho, que resultou numa redução na conversão final de metano para estes materiais.

Palavras-chave: Perovskitas, $\mathrm{LaMnO}_{3}$, Combustão, Metano.

\begin{abstract}
Perovskite-type Catalysts, $\mathrm{LaMnO}_{3}$, were synthesized by citrate and coprecipitation methods. Some of these materials was supported on $\gamma$-alumina or impregnated with Pd and/or Sn by wet impregnation, in $1 \%$ mass concentration. The catalysts were evaluated in the reaction of methane total combustion, and its activity measured by temperature programmed surface reaction (TPSR). The samples were heated to $800^{\circ} \mathrm{C}$ at a rate of $10^{\circ} \mathrm{C} /$ min under a flow rate of $50 \mathrm{~mL} /$ min of a mixture containing $\mathrm{CH}_{4}, \mathrm{O}_{2}$ and $\mathrm{N}_{2}$ at molar concentrations of $4 \%, 1 \%$ and $95 \%$, and $\mathrm{He}(50 \mathrm{~mL} / \mathrm{min})$. As a standard of comparison, was synthesized also a reference sample $\mathrm{PdO} / \gamma-\mathrm{Al}_{2} \mathrm{O}_{3}$. The samples were characterized by thermogravimetric analysis (TGA), X-ray diffraction (XRD) and had its surface area measured by BET. The samples containing palladium were further analyzed in temperature programmed oxidation reaction (TPO) in order to determine the stability of PdO phases present in these materials. All samples were active to the methane combustion reaction, and the materials from citrate route higher than the coprecipitated material. The addition of palladium, even in low concentrations, was positive for catalytic activity, unlike tin, that resulted in a reduction in the final methane conversion for these materials.
\end{abstract}

Keywords: perovskite, $\mathrm{LaMnO}_{3}$, Combustion, Methane.

\section{INTRODUÇÃO}


Atualmente, existem diversas fontes de produção de energia, como petróleo, biomassa, carvão e hidroeletricidade. A contrapartida destes processos é o impacto ambiental que eles geram [1,2]. Desta maneira, diversos estudos têm sido realizados com o objetivo de minimizar estes impactos, maximizando o potencial energético.

Neste cenário, a combustão do gás natural aparece como uma alternativa interessante, não só devido a sua abundância na natureza, mas também porque produz elevada energia por mol de $\mathrm{CO}_{2}$ gerado ao mesmo tempo que emite menos poluentes $[1,3]$.

$\mathrm{O}$ gás natural é composto principalmente por $\mathrm{CH}_{4} \mathrm{e}$ sua combustão, Equação 1, gera, além de $\mathrm{CO}_{2} \mathrm{e}$ $\mathrm{H}_{2} \mathrm{O}$, gases indesejados como NOx, SOx, CO, ainda que em quantidades inferiores aos combustíveis fósseis usuais $[1,4]$.

$$
\begin{aligned}
& \mathrm{CH}_{4}(\mathrm{~g})+2 \mathrm{O}_{2}(\mathrm{~g}) \rightarrow \mathrm{CO}_{2}(\mathrm{~g})+2 \mathrm{H}_{2} \mathrm{O}(\mathrm{g}) \\
& \Delta \mathrm{H}_{298}=-890 \mathrm{~kJ} / \mathrm{mol}
\end{aligned}
$$

Uma maneira de tornar esta combustão mais eficiente, reduzindo a níveis baixíssimos a emissão destes poluentes é a promoção da reação na presença de um catalisador adequado [5,6]. A combustão catalítica promove uma queima mais efetiva, reduzindo a geração de monóxido de carbono. Além disso, ela ocorre a temperaturas mais baixas que a combustão convencional $\left(1300^{\circ} \mathrm{C}\right.$ e $2000^{\circ} \mathrm{C}$ respectivamente) [7]. Esta redução na temperatura desfavorece termodinamicamente a formação de óxidos de nitrogênio.

Devido às condições severas em que a reação ocorre, o catalisador empregado deve apresentar elevada estabilidade térmica e ser ativo o suficiente para reduzir a temperatura de ignição do sistema [8]. Dentre os catalisadores conhecidos, destacam-se os materiais a base de metais nobres, como o paládio [8]. Entretanto, devido ao seu custo elevado, materiais ativos e mais econômicos têm sido propostos como alternativa ao uso destes metais $[9,10]$.

Estudos indicam que catalisadores a base de óxidos mistos, como as perovskitas, apresentam-se ativos e termoestáveis quando aplicadas a combustão do metano $[9,10]$.

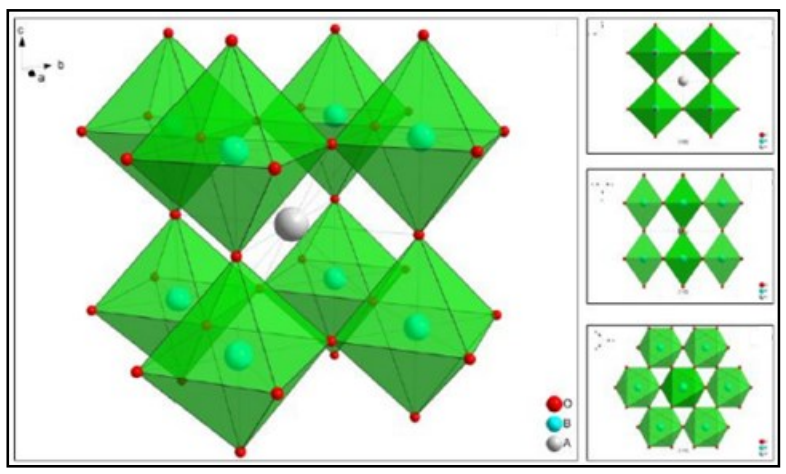

Figura 1: Estrutura perovskita ideal cúbica [8]

Perovskitas são compostos de estrutura molecular do tipo $\mathrm{ABO}_{3}$, onde o sítio $\mathrm{A}$ é um cátion alcalino, alcalino terroso ou terra rara nos estados de oxidação I, II, III ou IV e o sítio B um cátion de transição que, na maioria dos casos, assume estado de oxidação II, IV e V (Figuras 1 e 2). Estes cátions são contrabalanceados eletronicamente pelos ânions oxigênio na estrutura octaédrica. Os sítios A e B podem ser substituídos pordiversos elementos, possibilitando a formação de perovskitas de fórmulas bastante variadas [11]. A atividade é mais influenciada pela natureza do sítio $\mathrm{B}$, enquanto o sítio A tem ação majoritária na estabilidade do catalisador [12]. 


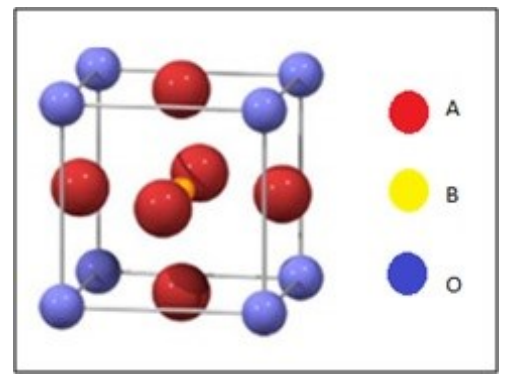

Figura 2: Célula unitária de uma estrutura peroviskita de fase cúbica $\mathrm{ABO}_{3}$ [13].

Neste trabalho, foram sintetizados catalisadores do tipo perovskita $\left(\mathrm{LaMnO}_{3}\right)$, através de duas rotas distintas: coprecipitação e citrato. Parte destes materiais catalíticos foi suportado em $\gamma$-alumina ou impregnados por via úmida com Pd e/ou Sn na concentração de 1\% em massa. Os catalisadores foram caracterizados por ATG, DRX e BET, testados na combustão total do metano por TPSR, de modo a avaliar a atividade catalítica destas amostras, e tiveram a sua estabilidade térmica avaliada por TPO.

\section{MATERIAIS E MÉTODOS}

\subsection{Preparação dos catalisadores}

\subsection{1 y-alumina}

A $\gamma$-alumina utilizada neste estudo foi obtida a partir da calcinação da bohemita (PURAL SB, SASOL) a $600^{\circ} \mathrm{C}$ por $3 \mathrm{~h}$ sob fluxo de ar $(50 \mathrm{~mL} / \mathrm{min})$.

\subsubsection{Citrato}

$\mathrm{La}\left(\mathrm{NO}_{3}\right)_{3} \cdot 6 \mathrm{H}_{2} \mathrm{O}$ e $\mathrm{Mn}\left(\mathrm{NO}_{3}\right)_{2} \cdot 4 \mathrm{H}_{2} \mathrm{O}$, foram adicionados a uma solução de ácido cítrico a $40^{\circ} \mathrm{C}$ por $20 \mathrm{~min}$ e sob agitação constante, numa proporção ácido cítrico/metal de 1,5:1. O nitrato de manganês foi o primeiro a ser adicionado e a solução resultante foi aquecida e mantida entre $60^{\circ} \mathrm{C}$ e $70^{\circ} \mathrm{C}$ por $1 \mathrm{~h}$. Adicionou-se então o nitrato de lantânio e aqueceu-se a solução, mantendo a sua temperatura entre $80^{\circ} \mathrm{C}$ e $90^{\circ} \mathrm{C}$ por mais $1 \mathrm{~h}$, até a formação da resina polimérica. $\mathrm{O}$ material foi então tratado termicamente a uma taxa de aquecimento de $5^{\circ} \mathrm{C} /$ min até $100^{\circ} \mathrm{C}$, permanecendo nesta temperatura por $1 \mathrm{~h}$ para, em seguida, ser aquecido até $300^{\circ} \mathrm{C}$ numa taxa de $10^{\circ} \mathrm{C} / \mathrm{min}$, permanecendo nesta temperatura por $2 \mathrm{~h} . \mathrm{O}$ sólido esponjoso formado foi macerado e calcinado a $800^{\circ} \mathrm{C}$, numa taxa de aquecimento de $10^{\circ} \mathrm{C} / \mathrm{min}$, por $4 \mathrm{~h}$ sob fluxo de ar sintético $(50 \mathrm{~mL} / \mathrm{min})$.

\subsubsection{Coprecipitação}

Soluções equimolares de $\mathrm{La}\left(\mathrm{NO}_{3}\right)_{3} \cdot 6 \mathrm{H}_{2} \mathrm{O}$ e $\mathrm{Mn}\left(\mathrm{NO}_{3}\right)_{2} \cdot 4 \mathrm{H}_{2} \mathrm{O}$, foram preparadas separadamente e misturadas num béquer, para então serem adicionados numa solução contendo excesso de hidróxido de amônio aquecida a $60^{\circ} \mathrm{C}$. A mistura foi mantida com $\mathrm{pH}$ igual a 8 e o aquecimento foi interrompido. O material foi conservado sob agitação por 30min. O precipitado formado foi filtrado com o auxílio de uma bomba de vácuo, lavado com água destilada e seco a $120^{\circ} \mathrm{C}$, por $12 \mathrm{~h}$ em estufa. $\mathrm{O}$ material foi então calcinado a $800^{\circ} \mathrm{C}$, numa taxa de aquecimento de $10^{\circ} \mathrm{C} / \mathrm{min}$, por $4 \mathrm{~h}$ sob fluxo de ar sintético $(50 \mathrm{~mL} / \mathrm{min})$.

\subsubsection{Perovskita suportada em Y-alumina}

Parte da perovskita sintetizada pela rota citrato foi misturada mecanicamente a $\gamma$-alumina. Preparou-se também por coprecipitação uma amostra da perovskita $\mathrm{LaMnO}_{3}$ suportada em $\gamma$-alumina, numa proporção perovskita/ $\gamma$-alumina de 70:30. Para tanto, utilizou-se a metodologia descrita anteriormente, apenas acrescetando-se a $\gamma$-alumina na solução de hidróxido de amônio utilizada no início do procedimento.

\subsubsection{Impregnação por via úmida}

Adicionou-se tolueno ao suporte desejado $\left(\gamma-\mathrm{Al}_{2} \mathrm{O}_{3}\right.$ ou $\left.\mathrm{LaMnO}_{3}\right)$ e manteve-se a solução resultante sob agitação constante por $24 \mathrm{~h}$, quando então foi adicionado o acetil acetonato de paládio $\left(\mathrm{Pd}\left(\mathrm{C}_{5} \mathrm{H}_{7} \mathrm{O}_{2}\right)_{2}\right)$ em quanti- 
dade suficiente para produzir um material com $1 \%$ em massa de Pd. A solução foi mantida sob agitação por mais $24 \mathrm{~h}$. $\mathrm{O}$ material foi aquecido a $110^{\circ} \mathrm{C}$ para a evaporação do solvente e então seco em estufa por $12 \mathrm{~h}$ sob a mesma temperatura. Posteriormente, calcinou-se a amostra resultante a $600^{\circ} \mathrm{C}$ por $3 \mathrm{~h}$ a uma taxa de aquecimento de $10^{\circ} \mathrm{C} / \mathrm{min}$, sob fluxo de ar sintético de $10 \mathrm{~mL} / \mathrm{min}$. Parte deste material foi impregnado com estanho a partir do mesmo procedimento, utilizando-se água destilada como solvente e cloreto de estanho $\left(\mathrm{SnCl}_{2}\right)$ como precursor.

\subsection{Caracterização}

\subsubsection{Difração de raios $X$ (DRX)}

As medidas de difração de raios $X$ foram conduzidas em um equipamento Shimadzu, modelo XRD-6000, utilizando-se a radiação $\mathrm{K} \alpha$ do $\mathrm{Cu}(\lambda=1,5418 \AA)$. Os difratogramas foram coletados num intervalo de $10^{\circ} \mathrm{a}$ $80^{\circ}$, com uma velocidade de varredura de $2 \%$ min para as amostras sem paládio e $0,2^{\circ} / \mathrm{min}$ para as amostras contendo paládio. Foram utilizados como referência os padrões obtidos no banco de dados JCDS.

\subsection{2 Área superficial - BET}

A área superficial dos catalisadores foi determinada num equipamento de adsorção de nitrogênio da Quanta Chrome Corporation, modelo NOVA -2000. As amostras foram desgaseificadas previamente a $200^{\circ} \mathrm{C}$ durante $1 \mathrm{~h}$.

\subsubsection{Análise termogravimétrica (TG)}

As análises foram realizadas num equipamento TGA-50 Shimadzu sob fluxo de ar sintético até $1000^{\circ} \mathrm{C}$ a $10^{\circ} \mathrm{C} / \mathrm{min}$.

\subsubsection{Reação superficial a temperatura programada (TPSR)}

As reações de oxidação total do metano foram realizadas em um reator de quartzo contendo $100 \mathrm{mg}$ de amostra misturados a $100 \mathrm{mg}$ de quartzo. As amostras foram aquecidas até $800^{\circ} \mathrm{C}$, a uma taxa de $10^{\circ} \mathrm{C} / \mathrm{min}$, sob um fluxo de $50 \mathrm{~mL} / \mathrm{min}$ de uma mistura contendo $\mathrm{CH}_{4}, \mathrm{O}_{2}$ e $\mathrm{N}_{2}$ com concentrações molares de $4 \%, 1 \%$ e $95 \%$, e $\mathrm{He}(50 \mathrm{~mL} / \mathrm{min})$. Os gases efluentes foram detectados utilizando-se um espectrômetro de massas quadrupolar, Balzers QMS-200.

\subsubsection{Oxidação a temperatura programada (TPO)}

A reação foi realizada em um reator de quartzo contendo $1000 \mathrm{mg}$ de amostra, em uma unidade multipropósito acoplada a um espectrômetro de massas quadrupolar da Balzers, modelo QMS 200.

Tratou-se as amostras a $600^{\circ} \mathrm{C}$ por $1 \mathrm{~h}$, com uma taxa de aquecimento de $10^{\circ} \mathrm{C} / \mathrm{min}$ e fluxo de uma mistura $5 \% \mathrm{O}_{2} / \mathrm{He}$ de $20 \mathrm{~mL} / \mathrm{min}$. Após o pré-tratamento, as amostras foram resfriadas até a temperatura ambiente e então aquecidas até $1000^{\circ} \mathrm{C}$, numa taxa de $10^{\circ} \mathrm{C} / \mathrm{min}$ e fluxo de $20 \mathrm{~mL} / \mathrm{min}$ de uma mistura $5 \%$ de $\mathrm{O}_{2}$ e $95 \%$ de He.

\section{RESULTADOS E DISCUSSÕES}

A partir da análise da Figura 3, é possível perceber que as perovskitas sintetizadas por ambos os métodos (citrato e coprecipitação) apresentaram um grau de cristalinidade bastante elevado. É possível indentifivar no difratograma picos característicos da fase perovskita $\mathrm{LaMnO}_{3}$ (JCDS 01-075-0440) em $2 \Theta=22,8^{\circ} ; 32,5^{\circ}$; $40,2^{\circ} ; 46,9^{\circ} ; 52,6^{\circ} ; 58,0^{\circ} ; 68,3^{\circ}$ e $77,8^{\circ}$ em todos os difratogramas. Para o $\mathrm{PdO} / \mathrm{LaMnO}_{3}$, nota-se ainda picos característicos do $\mathrm{PdO}$ (JCDS 00-006-0515), localizados em $2 \Theta=55^{\circ}$ e $72^{\circ}$ e no $\mathrm{PdO} / \mathrm{SnO} / \mathrm{LaMnO}_{3}$ nota-se um pico em $2 \Theta=29,87$, referente ao $\mathrm{SnO}$.

No difratograma da alumina pura, figura 4, foram identificados picos característicos da $\gamma-\mathrm{Al}_{2} \mathrm{O}_{3}$ (JCDS 00-047-1292) em $2 \Theta=31,6^{\circ} ; 37,1^{\circ} ; 39,1^{\circ} ; 45,8^{\circ} ; 60,7^{\circ}$ e $66,9^{\circ}$. No material impregnado com paládio por via úmida $(\mathrm{PdO} / \gamma-\mathrm{Al} 2 \mathrm{O} 3)$, além dos picos referentes a $\gamma-\mathrm{Al}_{2} \mathrm{O}_{3}$ citados anteriormente, são observados picos referentes ao óxido de paládio (JCDS 00-006-0515), localizados em $2 \Theta=34^{\circ} ; 55^{\circ}$ e $61^{\circ}$. Na amostra impregnada com estanho $\left(\mathrm{PdO} / \mathrm{Sn} / \gamma-\mathrm{Al}_{2} \mathrm{O}_{3}\right)$, nota-se ainda um ombro localizado em $2 \Theta=30$, referente ao $\mathrm{SnO}$. 
Nota-se a partir dos resultados apresentados que as técnicas de preparação utilizadas, tanto para síntese (coprecipitação e citrato) quanto para impregnação (via úmida), são adequadas para a formação dos catalisadores de interesse.

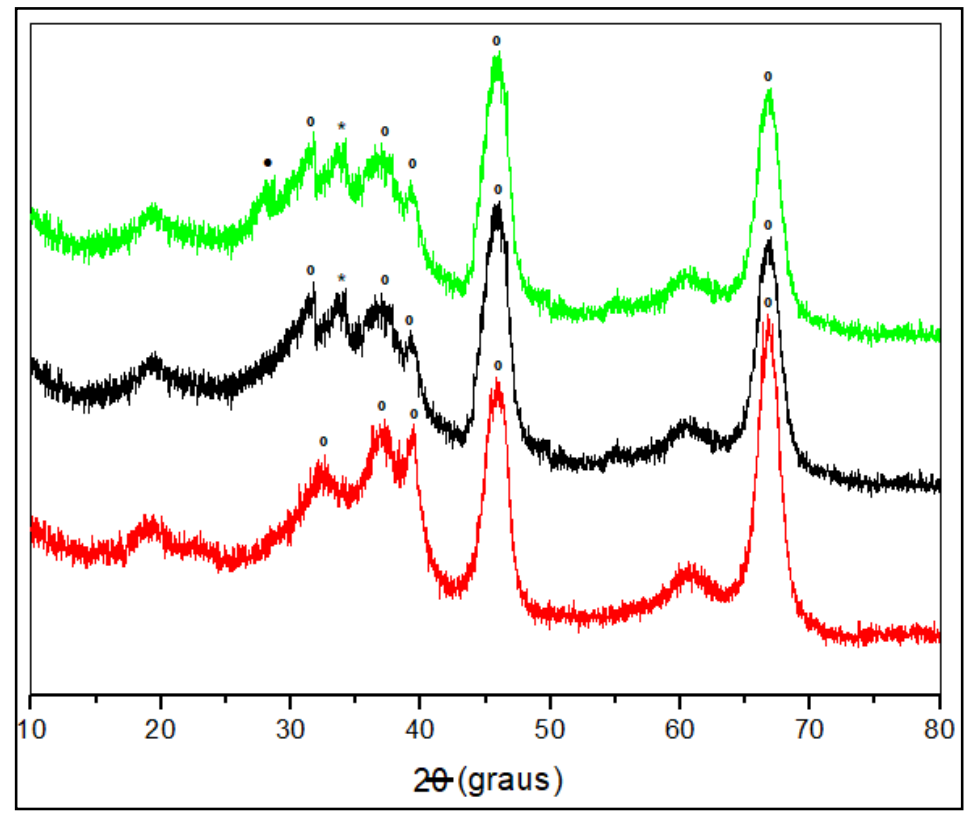

Figura 4: Difratograma das $\gamma$-aluminas. --- $\mathrm{PdO} / \mathrm{Al}_{2} \mathrm{O}_{3}{ }^{\prime}---\mathrm{Al}_{2} \mathrm{O}_{3},---\mathrm{PdO} / \mathrm{SnO} / \mathrm{Al}_{2} \mathrm{O}_{3}$. (o) $\mathrm{Al}_{2} \mathrm{O}_{3},\left({ }^{*}\right) \mathrm{PdO},(\bullet) \mathrm{SnO}$

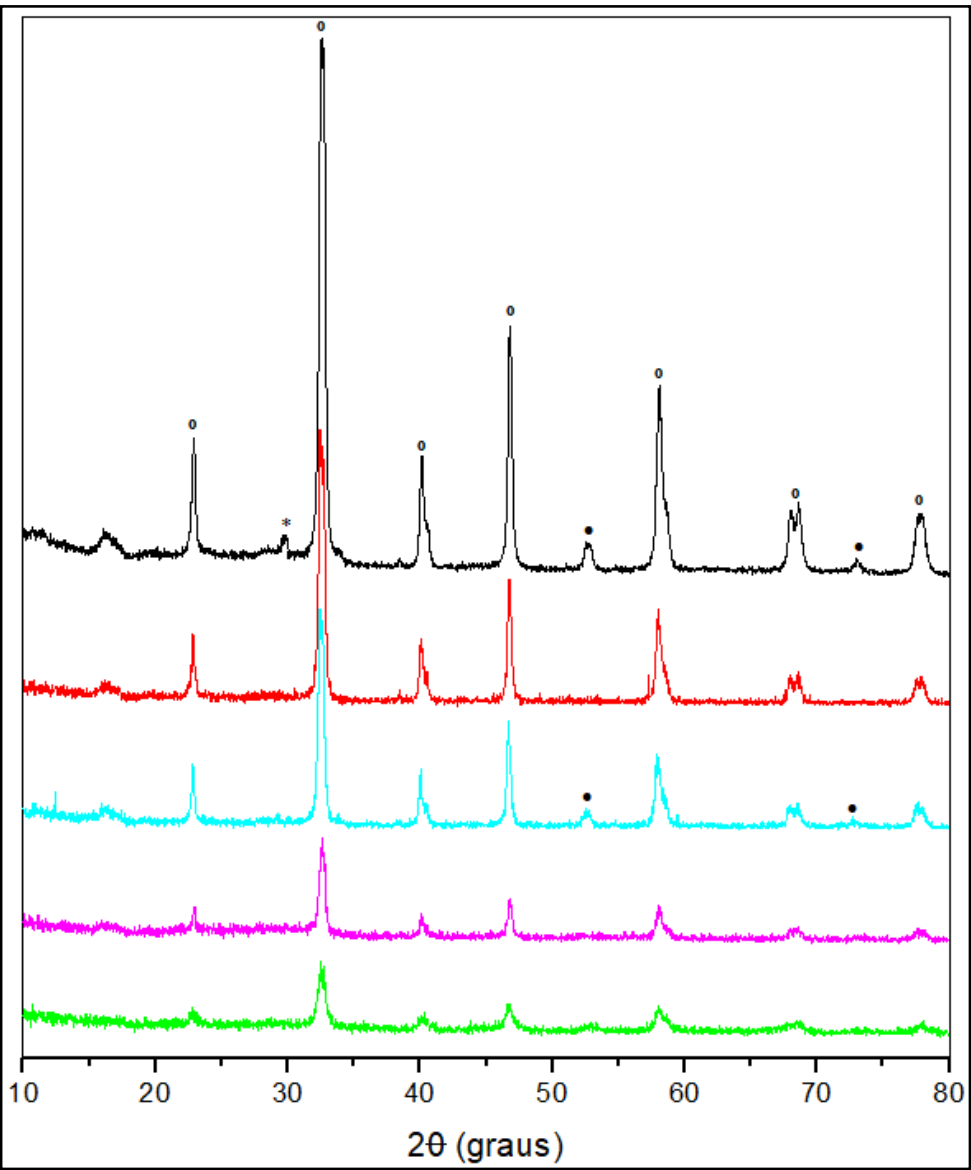

Figura 3: Difratograma das perovskitas. --- $\mathrm{LaMnO}_{3 \text { CIT }}$, --- $\mathrm{LaMnO}_{3} / \mathrm{Al}_{2} \mathrm{O}_{3 \text { coppt }}$, --- $\mathrm{LaMnO}_{3}$ coppt, --- $\mathrm{PdO} / \mathrm{LaMnO}_{3}$, -- $\mathrm{PdO} / \mathrm{SnOLaMnO}_{3}$. (o) $\mathrm{LaMnO}_{3},(*) \mathrm{SnO},(\bullet) \mathrm{PdO}$ 
O tamanho médio de cristalito (Tabela 1) foi determinado pela equação de Scherrer.

Tabela 1: Tamanho médio de cristalito

\begin{tabular}{l|l}
\hline CATALISADOR & TAMANHO MÉdIO DE CRISTALITO (Á) \\
\hline $\mathrm{LaMnO}_{3} \mathrm{CIT}$ & 165 \\
\hline $\mathrm{LaMnO} 3 / \gamma-\mathrm{Al}_{2} \mathrm{O}_{3} \mathrm{COPPT}$ & 116 \\
\hline $\mathrm{LaMnO} 3 \mathrm{COPPT}_{3}$ & 169 \\
\hline $\mathrm{PdO} / \mathrm{LaMnO} \mathrm{MIT}_{3} \mathrm{CIT}$ & 164 \\
\hline $\mathrm{PdO} / \mathrm{Sn} / \mathrm{LaMnO} \mathrm{MLIT}_{3} \mathrm{CIT}$ & 162 \\
\hline $\mathrm{PdO} / \gamma-\mathrm{Al}_{2} \mathrm{O}_{3}$ & 41 \\
\hline $\mathrm{PdO} / \mathrm{Sn} / \gamma-\mathrm{Al}_{2} \mathrm{O}_{3}$ & 43 \\
\hline$\gamma-\mathrm{Al}_{2} \mathrm{O}_{3}$ & 40 \\
\hline
\end{tabular}

Os resultados de BET (Tabela 2) apresentam baixa área superficial para as perovskitas. De acordo com a literatura, essa baixa área superficial já era esperada, visto que materiais do tipo perovskita são essencialmente fases de óxidos obtidas a elevadas temperaturas, necessárias para garantir um elevado grau de cristalinidade, mas que, no entanto, resultam num severo processo de sinterização e consequente redução da área superficial [14].

Os materiais contendo $\gamma$-alumina apresentaram elevadas áreas superficiais. Sabe-se que o suporte empregado é um importante componente do catalisador, já que este pode afetar profundamente na geração de espécies ativas para a promoção da reação [15]. Estes resultados estão associados a elevada área específica do suporte empregado, $\gamma-\mathrm{Al}_{2} \mathrm{O}_{3}$, que é um material amplamente utilizado por apresentar área superficial em torno de $200 \mathrm{~m}^{2} / \mathrm{g}$, contribuindo positivamente no desempenho de catalisadores a base de metais e/ou óxido de metais, aumentando a dispersão da fase ativa [16].

Pode-se verificar também que a rota de síntese utilizada, bem como a adição de paládio e/ou estanho nas concentrações adotadas, não influenciaram de maneira significativa a área superficial dos catalisadores.

Tabela 2: Área superficial

\begin{tabular}{|c|c|}
\hline CATALISADOR & ÁREA $\left(\mathrm{m}^{2} / \mathrm{g}\right)$ \\
\hline $\mathrm{PdO} / \gamma-\mathrm{Al}_{2} \mathrm{O}_{3}$ & 254,7 \\
\hline $\mathrm{LaMnO}_{3} / \gamma-\mathrm{Al}_{2} \mathrm{O}_{3} \mathrm{CIT}$ & 200,2 \\
\hline $\mathrm{LaMnO}_{3} \mathrm{CIT}$ & 12,9 \\
\hline $\mathrm{LaMnO}_{3} / \gamma-\mathrm{Al}_{2} \mathrm{O}_{3} \mathrm{COPPT}$ & 199,7 \\
\hline $\mathrm{LaMnO}_{3} \mathrm{COPPT}$ & 11,8 \\
\hline $\mathrm{PdO} / \mathrm{Sn} / \gamma-\mathrm{Al}_{2} \mathrm{O}_{3}$ & 214,7 \\
\hline $\mathrm{PdO} / \mathrm{LaMnO}_{3} \mathrm{CIT}$ & 12,0 \\
\hline $\mathrm{PdO} / \mathrm{Sn} / \mathrm{LaMnO}_{3} \mathrm{CIT}$ & 13,9 \\
\hline
\end{tabular}

De acordo com a análise de TG (Figura 5), pode-se perceber que, ao fim do aquecimento, restam apenas $55 \%$ da massa inicial utilizada. Isso significa que $45 \%$ da massa do sólido obtido antes da calcinação trata-se de matéria orgânica e água oriundas das etapas de síntese que, após todas as etapas de decomposição, foram desprendidas do material. Para ambas amostras, notam-se perfis de decomposição bastante semelhantes.

A primeira etapa de decomposição, que tem início um pouco abaixo de $100^{\circ} \mathrm{C}$, está diretamente relacionada com a dessorção de água fisissorvida remanescente do material pré-calcinado, seguido pela oxidação de espécies citrato livres, resultando na liberação de monóxido de carbono e água. A segunda etapa, em torno de $220^{\circ} \mathrm{C}$ está associada a calcinação dos citratos de La-Mn, que resulta na formação de uma mistura de dióxido de manganês $\left(\mathrm{MnO}_{2}\right)$ e $\mathrm{La}_{2} \mathrm{O}_{2} \mathrm{CO}_{3}$. Esta mistura é então convertida a dióxido de carbono $\left(\mathrm{CO}_{2}\right)$ e $\mathrm{LaMnO}_{3}$, na terceira etapa de decomposição, em torno de $270{ }^{\circ} \mathrm{C}$. 
A partir dos resultados obtidos, verifica- que não ocorre variação significativa de massa acima de $750^{\circ} \mathrm{C}$. Desta maneira, optou-se por calcinar estes materiais a temperaturas um pouco superiores, estabelecendo por segurança, temperatura de calcinação igual a $800^{\circ} \mathrm{C}$.

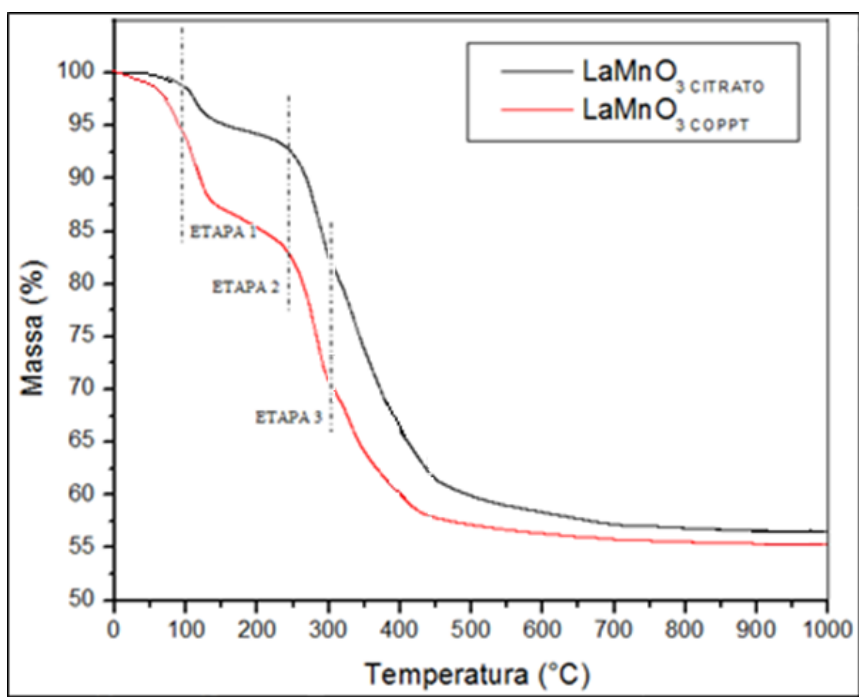

Figura 5: Análise termogravimétrica

Todos os catalisadores sintetizados neste trabalho apresentaram valores de conversão acima de $70 \%$, o que indica que são ativos para a combustão do metano. No entanto, os catalisadores impregnados com $\mathrm{Sn}$ apresentaram considerável queda de conversão a partir de $600^{\circ} \mathrm{C}$. O desempenho inferior apresentado pelos catalisadores contendo estanho pode estar associada a um aumento do tamanho das partículas de paládio presente nestes materiais, o que reduz a sua mobilidade e, consequentemente, a sua eficiência na combustão catalítica [17]. Além disso, o estanho apresenta baixo ponto de fusão $\left(232^{\circ} \mathrm{C}\right)$, de modo que a decomposição do óxido de estanho acima de $600^{\circ} \mathrm{C}$ pode ter levado a fusão do $\mathrm{Sn}$ metálico, com recobrimento da superfície do catalisador e afogamento dos sítios de $\mathrm{Pd}$.

As temperaturas de isoconversão $\left(\mathrm{T}_{20 \%}\right)$ dos materiais estudados são apresentadas na Tabela 3. Os catalisadores suportados em $\gamma$-alumina apresentaram menor temperatura de isoconversão e maior conversão final quando comparados às perovskitas puras. Sabe-se que as reações ocorrem principalmente na superfície dos catalisadores, de modo que a atividade destes materiais está intimamente ligada à sua área superficial. Desta maneira, os resultados obtidos por TPSR confirmam informações descritas na literatura [18] e estão de acordo com os resultados obtidos por BET, que indicaram áreas superficiais quase vinte vezes maiores para os catalisadores suportados em $\gamma$-alumina do que os catalisadores a base de perovskita.

Do ponto de vista da rota de preparação, nota-se que os materiais sintetizados pela rota citrato mostraram-se superiores tanto do ponto de vista da temperatura de isoconversão quanto da conversão final. Sabe-se que catalisadores preparados a partir da rota citrato apresentam maior homogeneidade e dispersão do que os materiais sintetizados por coprecipitação [19].

A adição de paládio, como esperado, reduziu significativamente a temperatura de isoconversão da perovskita, alcançando também uma conversão bastante elevada, próxima a 100\%. Isso se deve a elevada atividade catalítica do paládio, já que é sabido que metais nobres apresentam maior atividade que óxidos mistos de metais, como as perovskitas [20].

$\mathrm{O}$ catalisador de referência, $\mathrm{PdO} / \gamma-\mathrm{Al} 2 \mathrm{O} 3$, foi o que apresentou melhor atividade para a combustão do metano. Este material é um dos mais estudados para este tipo de reação e o seu desempenho está associado a já citada excelente atividade catalítica do óxido de paládio com a elevada área superficial específica da $\gamma$ alumina. Esta interação do metal com o suporte reduz, ainda, a degradação térmica sofrida pelo PdO a altas temperaturas [21].

A adição de estanho ao catalisador $\mathrm{PdO} / \mathrm{LaMnO} 3 \mathrm{CIT}$ levou a uma redução na conversão final da reação. Nota-se que a conversão atinge um máximo e então começa a cair, indo de $78 \%$ de conversão do metano em $634^{\circ} \mathrm{C}$ para $18 \%$ em $800^{\circ} \mathrm{C}$. Este fenômeno pode ser consequência da fusão do estanho e consequente afogamento do óxido de paládio no catalisador, sugerindo que a adição de $\mathrm{Sn}$, com a rota de preparação e teor utilizados, prejudica a estabilidade deste material a altas temperaturas, tendo a sua eficiência limitada a 
$634^{\circ} \mathrm{C}$. No que diz respeito a temperatura de isoconversão, não houve alteração perceptível após a impregnação de Sn nesta amostra.

A adição de estanho ao catalisador $\mathrm{PdO} / \gamma-\mathrm{Al}_{2} \mathrm{O}_{3}$ levou a uma redução na conversão final da reação, porém não tão drástica quanto no caso anterior. É possível visualizar na curva de conversão do metano sobre este material uma desaceleração no aumento da conversão, de modo que esta fica estagnada em $72 \%$ entre $656^{\circ} \mathrm{C}$ e $746^{\circ} \mathrm{C}$, quando então volta a crescer, atingindo $77 \%$ ao final da reação. A conversão do metano sobre o $\mathrm{PdO} / \mathrm{Sn} / \gamma-\mathrm{Al}_{2} \mathrm{O}_{3}$ começa a ser afetada na mesma faixa de temperatura que ocorre a queda de conversão para o catalisador $\mathrm{PdO} / \mathrm{LaMnO}_{3} \mathrm{CIT}$ (aproximadamente $600^{\circ} \mathrm{C}$ ), o que reforça a ideia de que este fenômeno esteja relacionado a fusão do estanho e consequente afogamento do óxido de paládio.

Os resultados apresentados estão de acordo com as informações da literatura, que relatam elevada atividade catalítica de perovskitas para a reação de combustão de metano, porém inferiores aos resultados alcançados por materiais a base de óxido de paládio (PdO), que por sua vez têm custo mais elevado [22].

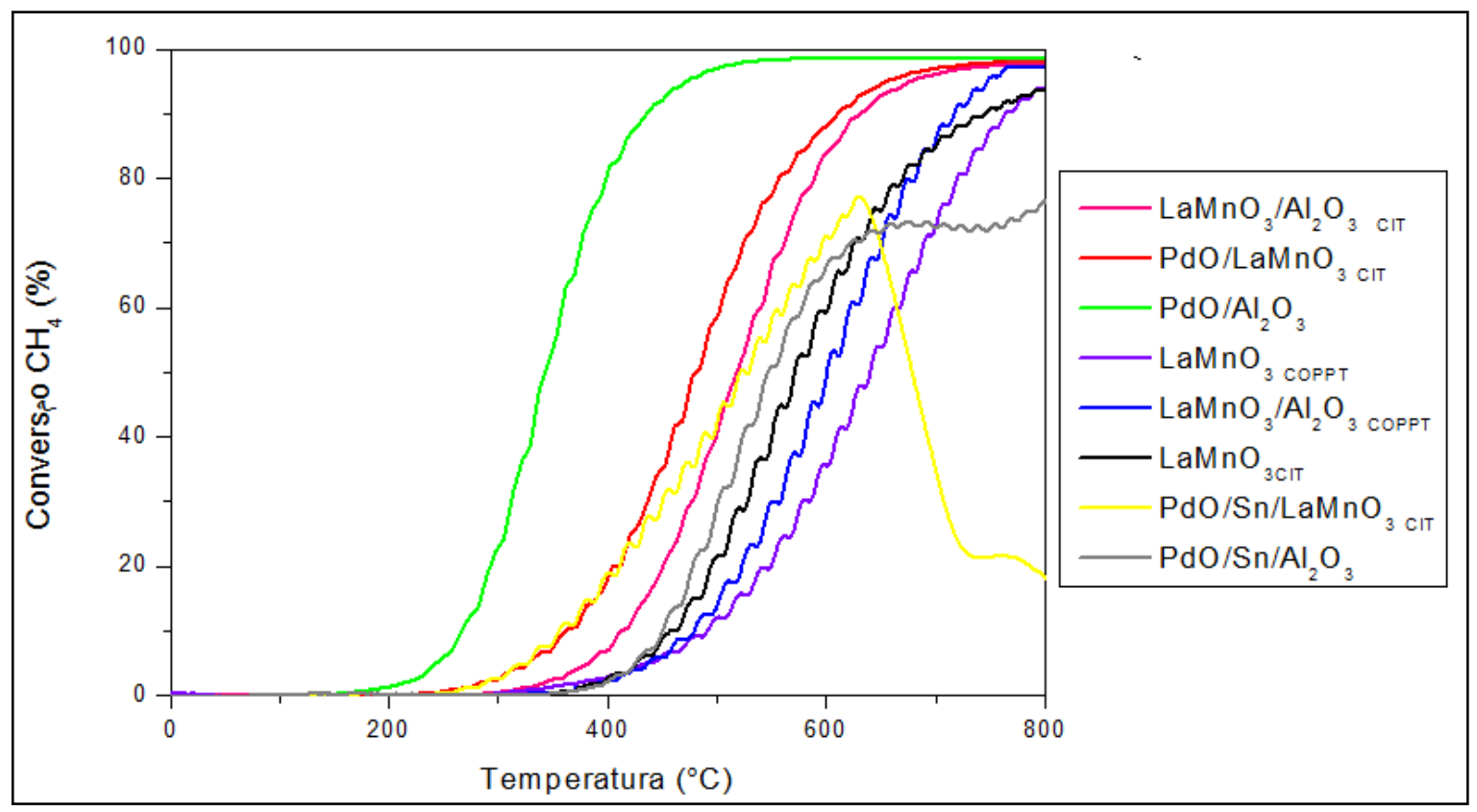

Figura 6: Perfis de TPSR dos catalisadores

Tabela 3: Temperatura de isoconversão $\left(\mathrm{T}_{20 \%}\right)$ e conversão final dos catalisadores

\begin{tabular}{l|l|l}
\hline CATALISAdOR & $\mathbf{T}_{\mathbf{2 0} \%}\left({ }^{\circ} \mathbf{C}\right)$ & CONVERSÃO (\%) \\
\hline $\mathrm{PdO} / \gamma-\mathrm{Al}_{2} \mathrm{O}_{3}$ & 291 & 99 \\
\hline $\mathrm{LaMnO}_{3} / \gamma-\mathrm{Al}_{2} \mathrm{O}_{3} \mathrm{CIT}$ & 449 & 97 \\
\hline $\mathrm{LaMnO}_{3} \mathrm{CIT}$ & 494 & 97 \\
\hline $\mathrm{LaMnO} / \gamma-\mathrm{Al}_{2} \mathrm{O}_{3} \mathrm{COPPT}$ & 521 & 94 \\
\hline $\mathrm{LaMnO} \mathrm{COPPT} \mathrm{COP}$ & 547 & 94 \\
\hline $\mathrm{PdO} / \mathrm{Sn} / \gamma-\mathrm{Al}_{2} \mathrm{O}_{3}$ & 477 & 77 \\
\hline $\mathrm{PdO} / \mathrm{LaMnO}{ }_{3} \mathrm{CIT}$ & 409 & 98 \\
\hline $\mathrm{PdO} / \mathrm{Sn} / \mathrm{LaMnO}_{3} \mathrm{CIT}$ & 409 & 18
\end{tabular}

A conversão do óxido de paládio em paládio metálico durante a oxidação do metano, exerce um efeito considerável na reatividade do catalisador [8]. A estabilidade do óxido é desfavorecida termodinamicamente com o aumento da temperatura do sistema, de modo que o $\mathrm{PdO}$ é convertido em $\mathrm{Pd}^{\circ}$ [20].

Para as perovskitas puras, as alterações no sinal de oxigênio estão relacionadas a alteração do estado de oxidação do manganês presente nestas amostras. Durante o aquecimento, observa-se o Mn variando de 
Mn4+ para Mn3+ e, no resfriamento, ocorre a reação inversa. A elevada atividade de catalisadores do tipo $\mathrm{LaMnO}_{3}$ é atribuída a presença do manganês nestes dois estados de oxidação distintos, resultando num constante excesso de oxigênio na estrutura [12].

A atividade destes materiais está intimamente associada a esta alteração no estado de oxidação das suas respectivas fases ativas.

Nota-se, portanto, uma relação entre os resultados de TPO e TPSR dos catalisadores estudados (Tabela 5). A tendência é que nestes materiais quanto maior a diferença entre as temperaturas de decomposição e reoxidação $(\Delta \mathrm{T})$, melhor o desempenho destes catalisadores frente a combustão catalítica do metano. Estes resultados sugerem que as análises de TPO também podem ser utilizadas como parâmetro para a comparação da atividade de catalisadores frente a combustão catalítica do metano.

Tabela 4: Temperaturas de decomposição e reoxidação e $\Delta \mathrm{T}$ dos catalisadores.

\begin{tabular}{|c|c|c|c|}
\hline CATALISADOR & $\mathrm{T}_{\mathrm{DECOMPOSIÇÃO}}$ * $\left({ }^{\circ} \mathrm{C}\right)$ & $T_{\text {REOXIDAÇÃO }}{ }^{*}\left({ }^{\circ} \mathrm{C}\right)$ & $\Delta \mathbf{T}^{*}\left({ }^{\circ} \mathrm{C}\right)$ \\
\hline $\mathrm{LaMnO}_{3}$ CIT & 677 & 912 & 235 \\
\hline $\mathrm{LaMnO}_{3} \mathrm{COPPT}$ & 812 & 908 & 96 \\
\hline $\mathrm{PdO} / \mathrm{Al}_{2} \mathrm{O}_{3}$ & 885 & 530 & 355 \\
\hline $\mathrm{PdO} / \mathrm{Sn} / \gamma-\mathrm{Al}_{2} \mathrm{O}_{3}$ & 824 & 566 & 258 \\
\hline $\mathrm{PdO} / \mathrm{LaMnO}_{3} \mathrm{CIT}$ & 930 & 597 & 333 \\
\hline $\mathrm{PdO} / \mathrm{Sn} \mathrm{LaMnO}_{3} \mathrm{CIT}$ & 852 & 640 & 212 \\
\hline
\end{tabular}

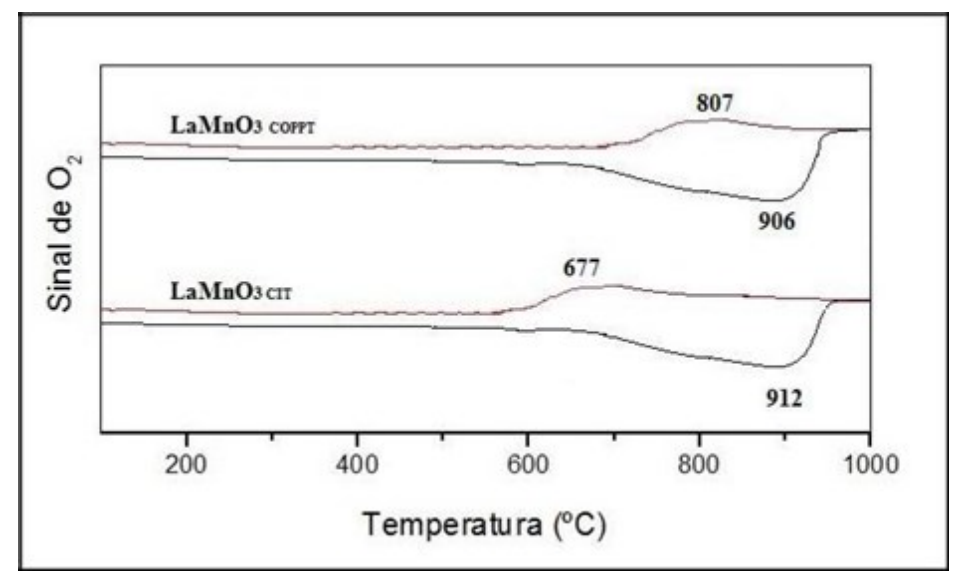

Figura 7: Perfis de TPO das perovskitas puras. (--)Aquecimento, (--)Resfriamento

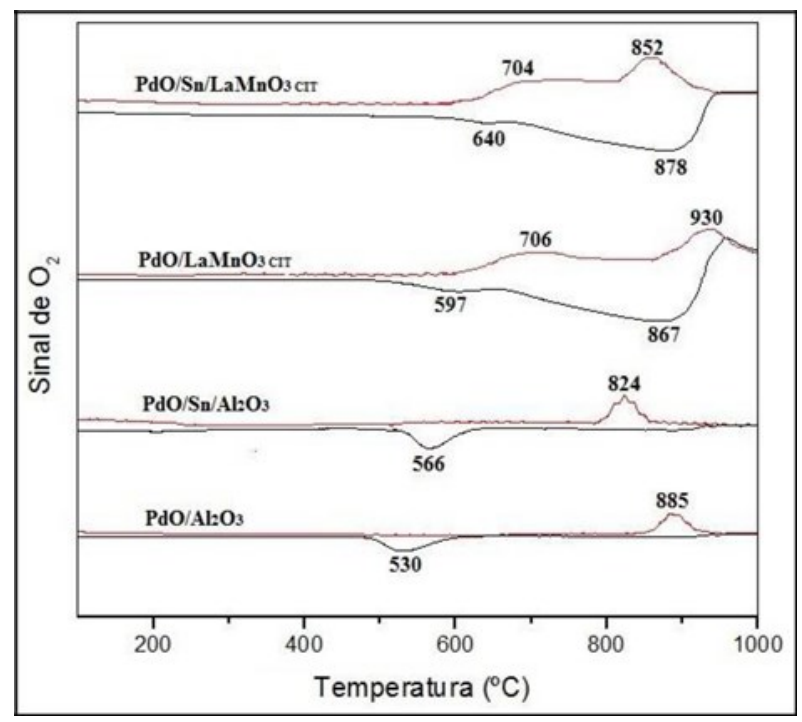

Figura 8: Perfis de TPO para as amostras contendo Pd. (--)Aquecimento, (--)Resfriamento 


\begin{tabular}{l|l|l|l}
\hline CATALISADOR & $\mathbf{T}_{\mathbf{2 0} \%}\left({ }^{\circ} \mathbf{C}\right)$ & CONVERSÃO (\%) & $\Delta \mathbf{T}^{*}\left({ }^{\circ} \mathbf{C}\right)$ \\
\hline $\mathrm{LaMnO}_{3} \mathrm{CIT}$ & 494 & 97 & 235 \\
\hline $\mathrm{LaMnO}{ }_{3} \mathrm{COPPT}$ & 547 & 94 & 96 \\
\hline $\mathrm{PdO} / \gamma-\mathrm{Al}_{2} \mathrm{O}_{3}$ & 291 & 99 & 355 \\
\hline $\mathrm{PdO} / \mathrm{Sn} / \gamma-\mathrm{Al}_{2} \mathrm{O}_{3}$ & 477 & 77 & 258 \\
\hline $\mathrm{PdO} / \mathrm{LaMnO} \mathrm{CIT}_{3}$ & 409 & 98 & 333 \\
\hline $\mathrm{PdO} / \mathrm{Sn} \mathrm{LaMnO}_{3} \mathrm{CIT}$ & 409 & 18 & 212 \\
\hline
\end{tabular}

\section{CONCLUSÕES}

As rotas de síntese empregadas foram eficientes para a formação das perovskitas desejadas com tamanho de cristalito similares. A perovskita sintetizada pela rota citrato, no entanto, apresentou melhor atividade catalítica. A adição de paládio, mesmo em baixa concentração, melhorou consideravelmente o desempenho destes materiais, bem como a presença de $\gamma$-alumina que melhorou bastante a dispersão da fase ativa e, consequentemente, a atividade frente a combustão do metano. A adição de $\mathrm{Sn}$ reduziu a atividade dos catalisadores, devido ao afogamento do Pd nestas amostras, de modo que estes materiais não são indicados para combustão em altas temperaturas. A relação entre os resultados de TPO e TPSR dos catalisadores impregnados com Pd sugere que as análises de TPO podem ser utilizadas como padrão de comparação entre as atividades destes materiais frente a reação de combustão catalítica do metano. Desta maneira, quanto maior a diferença entre as temperaturas de redução e reoxidação do paládio presente nestas amostras, menor será a sua temperatura de ignição.

\section{AGRADECIMENTOS}

Ao CAPES e CNPq pelo suporte financeiro e ao LABCAT pelas análises de TG e BET.

\section{BIBLIOGRAFIA}

[1] JODLOWSKI, P. J. "In situ spectroscopic studies of methane catalytic combustion over Co, Ce, and Pd mixed oxides deposited on a steel surface", Journal of Catalysis, v. 350, pp. 1-12, Jun. 2017.

[2] MERCEAU, E., CHE, M., TATIBOUET, J. M. "Influence of chlorine ions in $\mathrm{Pt} / \mathrm{A}_{2}{ }_{2} \mathrm{O}_{3}$ catalysts for methane total oxidation”, Catalysis Today, v. 29, pp. 1-4, May 1996.

[3] ZHAO, Z., WANG, B., MA, J., et al., "Catalytic combustion of methane over $\mathrm{Pd} / \mathrm{SnO}_{2}$ catalysts". Chinese Journal of Catalysis, v. 38, pp. 1322-1329, Aug. 2017.

[4] DING, Y., WANG, S., ZHANG, L., et al., "A facile method to promote $\mathrm{LaMnO}_{3}$ perovskite catalyst for combustion of methane”, Catalysis Communication, v. 97, pp. 88, Jul. 2017.

[5] WANG, L., XIE, H., WANG, X., et al., "Preparation of $\mathrm{LaMnO}_{3}$ for catalytic combustion of vinyl chloride", Chinese Journal of Catalysis, v. 38, pp. 1406-1412, Aug. 2017.

[6] XU, P., ZHAO, X., ZHANG, X., et al., "Three-dimensionally ordered macroporous $\mathrm{LaMnAl}_{11} \mathrm{O}_{19}$ supported Pd nanocatalysts highly active for methane combustion”, Molecular Catalysis, v. 439, pp. 200-210, Oct. 2017.

[7] XU, P., WU, Z., DENG, J., et al., "Catalytic performance enhancement by alloying Pd with Pt on ordered mesoporous manganese oxide for methane combustion”, Chinese Journal of Catalysis, v. 38, pp 92-105, Jan. 2017.

[8] BAYLET, A., ROYER, S., MARÉCOT, P., et al., "High catalytic activity and stability of Pd doped hexaaluminate catalysts for the $\mathrm{CH}_{4}$ catalytic combustion”, Applied Catalysis B: Enviromental, v. 77, pp. 237-247, Jan. 2008.

[9] SHATERIAN, M., ENHESSARI, M., RABBANI, D., et al., " Synthesis, characterization and photocatalytic activity of $\mathrm{LaMnO}_{3}$ nanoparticles”, Applied Surface Sciences, v. 318, pp 213-217, Nov. 2014.

[10] SAY, Z., DOGAC, M., VOVK, E. I., et al., "Palladium doped perovskite-based NO oxidation catalysts: The role of $\mathrm{Pd}$ and B-sites for NOx adsorption behavior via in-situ spectroscopy", Applied Catalysis B : Enviromental, v. 154-155, pp 51-61, Aug. 2014. 
[11] LU, Y., DAI, Q., WANG, X. "Catalytic combustion of chlorobenzene on modified $\mathrm{LaMnO}_{3}$ catalysts", Catalysis Communications, v. 54, pp. 114-117, Sep. 2014.

[12] SUPELANO, G. I., BARÓN-GONZÁlEZ, A., SANTOS, A. S., et al., "Effect of Mg addition on $\mathrm{LaMnO}_{3}$ ceramic system", Journal of Materials Research and Technology, v. 244, pp. 2288, Aug. 2017.

[13] SHAFEIE, S. Structure and property investigations of the $\mathrm{La}_{2} \mathrm{Co}_{1+z}\left(\mathrm{Ti}_{1-\mathrm{x}} \mathrm{Mg}_{\mathrm{x}}\right)_{1-\mathrm{z}} \mathrm{O}_{6}$ perovskite system, Tese de D.Sc., Stockholms Universitet, Estocolmo, Suécia, 2011.

[14] SILVA, P. R. N., "Emprego de óxidos tipo perovskite nas oxidações do propano e CO", Química Nova, v. 27, pp. 35-41, Sep. 2004.

[15] WANG, Y., WEI, Q., HUANG, Y. "Preparation and adsorption properties of the biomimetic gamaalumina", Materials Letters, v. 157, pp. 67-69, Oct. 2015.

[16] ERCOLINO, G., KARIMI, S., STELMACHOWSKI, P., et al., "Catalytic combustion of residual methane on alumina monoliths and open cell foams coated with $\mathrm{Pd} / \mathrm{Co}_{3} \mathrm{O}_{4}$ ", Chemical Engineering Journal, v. 326, pp. 339-349, Oct. 2017.

[17] BERDIYOROV, G. R., MADJET, M. E., EL-MELLOUHI, F. "Improved electronic transport properties of tin-halide perovskites", Solar Energy Materials and Solar Cells, v. 1701, pp. 8-12, Oct. 2017.

[18] ROS, S., BARBOSA-COUTINHO, E., SCHWAAB, M., et al., "Modeling the effects of calcination conditions on the physical and chemical properties of transition alumina catalysts", Materials Characterization, v.80, pp. 50-61, Jun. 2013.

[19] CIMINO, S., BENEDETTO, A., PIRONE, R., et al., " $\mathrm{CO}, \mathrm{H}_{2}$ or $\mathrm{C}_{3} \mathrm{H}_{8}$ assisted catalytic combustion of methane over supported $\mathrm{LaMnO}_{3}$ monoliths", Catalysis Today, v. 83, pp. 33-43, Aug. 2003.

[20] PETROVIC, S., KARANOVIC, L., STEFANOV, P. K., et al., "Catalytic combustion of methane over Pd containing perovskite type oxides", Applied Catalysis B : Enviromental, v. 58, pp. 133-141, Jun. 2005.

[21] BOUKHA, Z., FITIAN, L., LÓPEZ-HARO, M., et al., "Influence of the calcination temperature on the nano-structural properties, surface basicity, and catalytic behavior of alumina-supported lanthana samples", Journal of catalysis, v.272, pp. 121-130, May, 2010.

[22] YANG, J., GUO, Y. "Nanostructured perovskite oxides as promising substitutes of noble metals catalysts for catalytic combustion of methane", Chinese Chemical Letters, 2017. Disponível em: https://doi.org/10.1016/j.cclet.2017.09.013. 